\title{
Cancer rehabilitation and palliative care-two important parts of comprehensive cancer care
}

\author{
Richard Crevenna ${ }^{1}$
}

Received: 29 June 2015 / Accepted: 28 September 2015 / Published online: 6 October 2015

(C) Springer-Verlag Berlin Heidelberg 2015

In this issue of Supportive Care in Cancer, Julie K. Silver et al. focus on cancer rehabilitation and palliative care delineating their respective roles in comprehensive oncology care [1]. The authors describe how cancer rehabilitation and palliative care are quite similar in goal setting, namely to improve physical, cognitive, psychological, and social aspects of quality of life in cancer patients - both specialties prioritizing goalconcordant care through identification of patient and family preferences and values. So both - cancer rehabilitation and palliative care - are able to improve cancer-related symptoms or cancer treatment-related side effects leading not only to (1) improve quality of life of cancer patients and cancer survivors but also to (2) improve healthcare efficiencies. As palliative care is, cancer rehabilitation is an interdisciplinary team process and an individually planned targeted process with the patient and family as active members within the team. Exercise - as a modality typical for cancer rehabilitationcan even help patients where palliative rehabilitation goals are predominant to live longer and independent of others help [2]. Nevertheless, cancer rehabilitation and palliative care are using different specialized skills and approaches in the delivery of care. Typically, palliative care teams focus on using patient and family communication to determine their goals of care, but rehabilitation teams focus more on functional deficits and their resulting impact on disability and quality of life. The authors conclude that further research in cancer rehabilitation and palliative care would support better integration of

Richard Crevenna

richard.crevenna@meduniwien.ac.at

1 Department of Physical Medicine and Rehabilitation, Medical University of Vienna, Waehringer Guertel 18-20,

A-1090 Vienna, Austria both into high-quality oncology care. Furthermore, they emphasize earlier integration of cancer rehabilitation and palliative care into the oncology care continuum because this would significantly improve the quality of patient-centered programs.

At the Comprehensive Cancer Centre (CCC) of the Medical University of Vienna, Austria, there has been implemented a so-called "CCC-Platform for Side effectsManagement, Supportive Care \& Rehabilitation" to improve quality of care. Such a "CCC-Platform" has the goal to integrate all different medical disciplines involved in a cancerspecific issue (for example, side effect-management, supportive care and rehabilitation) under the umbrella of the CCC. This new platform will integrate different existing services like an "Outpatient Clinic for Cancer Rehabilitation," a "CCC-Tumorboard for Cancer Rehabilitation," an "Outpatient Clinic for Medical Exercise and Training," a "CCC-Platform Sexual Medicine," the "Cancer Care" service, services for "Nutrition" and "Lifestyle modification," a "CCC-Platform for Management of the Extravasation of Anti-Neoplastic Agents," and different services for the application of "Complementary Medicine in Cancer Patients." Furthermore, there will be a close cooperation with the "Inpatient Clinic for Palliative Medicine." The members and the services of this CCC-Platform for Side effectsManagement, Supportive Care \& Rehabilitation have a definitive goal, namely to improve bodily, cognitive, mental, and social functions of cancer patients, to improve their quality of life, and to increase their (social) participation. Furthermore, this CCC-Platform for Side effects-Management, Supportive Care \& Rehabilitation should enhance adherence rates in cancer treatment due to integrated and better management of side effects (like fatigue, pain, polyneuropathy, loss of muscle mass and decreased muscular strength and endurance capacity, nutritional deficits, mucositis, hand-foot syndrome, 
extravasation of anti-neoplastic agents, lymphedema, incontinence, sexual dysfunctions, cognitive deficits, dysthymia) of cancer and its treatment (oncological therapies). This should not only improve quality of life of cancer patients and survivors but also their outcomes and survival due to better adherence during curative cancer treatment. This interdisciplinary CCC-Platform which (at the moment) is located at the Department of Physical Medicine and Rehabilitation can be used by specialists of all involved disciplines. It operates from 8 am to $4 \mathrm{pm}, 5$ days a week to ensure uninterrupted access to the services. The delivery of care should be efficient, avoiding rework, redundancy, or work-around solutions, and patients should not waste time waiting too long to get an appointment. Early referral to rehabilitation and palliative care services is paramount to maximize the quality of care offered to these patients, and with the help of this platform, an advanced access strategy can be offered. In our opinion, this platform is a modern way to better integrate cancer rehabilitation and palliative care services into the whole oncology care continuum, although we assume that this new pilot project will need time to be fully understood by all (colleagues, patients, caregivers, families). For the future, research to evaluate the impact of this upcoming CCC-Platform on the quality of life of oncology patients is planned.

It is evident that many cancer survivors would benefit from cancer rehabilitation, and that palliative care is a proven way to help cancer patients and their families to a better life. Nevertheless, an underutilization of both services - cancer rehabilitation and palliative care-remains and is well documented [3-7]. Therefore, healthcare professionals should be encouraged to better understand and to recommend (more often and earlier!) cancer rehabilitation and palliative care services to colleagues, patients, and their families in order to improve access to quality care. The use of platforms integrating the different services under the "umbrella" of an institution like the CCC could be one possibility to reach these goals.
Nevertheless, further activities (for example, best quality of care, best clinical practice and research, best clinical teaching) involving all relevant "players" to enhance the value of cancer rehabilitation and palliative care services within the population are urgently needed.

\section{Compliance with ethical standards}

Conflict of interest The author declares that he has no competing interests.

\section{References}

1. Silver JK, Raj VS, FU JB, Wisotzky EM, Smith SR, Kirch RA (2015) Cancer rehabilitation and palliative care: critical components in the delivery of high-quality oncology services. Support Care Cancer XXX

2. Crevenna R (2013) From neuromuscular electrical stimulation and biofeedback-assisted exercise up to triathlon competitions - regular physical activity for cancer patients in Austria. Eur Rev Aging Phys Act 10(1):53-55

3. Silver JK, Baima J, Mayer RS (2013) Impairment-driven cancer rehabilitation: an essential component of quality care and survivorship. CA Cancer J Clin 63(5):295-317. doi:10.3322/caac.21186

4. Cheville AL, Troxel AB, Basford JR, Kornblith AB (2008) Prevalence and treatment patterns of physical impairments in patients with metastatic breast cancer. J Clin Oncol 26(16):26212629. doi:10.1200/JCO.2007.12.3075

5. Pergolotti M, Deal AM, Lavery J, Reeve BB, Muss HB (2015) The prevalence of potentially modifiable functional deficits and the subsequent use of occupational and physical therapy by older adults with cancer. J Geriatr Oncol. doi:10.1016/j.jgo.2015.01.004

6. Institute of Medicine (2013) Delivering high-quality cancer care: charting a new course for a system in crisis. National Academies Press, Washington DC

7. Bruera E, Hui D (2012) Conceptual models for integrating palliative care at cancer centers. J Palliat Med 15(11):1261-1269. doi:10.1089/ jpm.2012.0147 